\title{
Evaluation of wound healing activity of Hibiscus rosa sinensis linn in albino rats
}

\author{
Monil Yogesh Neena Gala*, Swanand S. Pathak
}

Department of Pharmacology, Jawaharlal Nehru Medical College, DMIMS, Sawangi, Meghe, Wardha, Maharashtra, India

Received: 22 March 2019

Accepted: 11 April 2019

Revised: 03 May 2019

\section{*Correspondence to:}

Dr. Monil Yogesh Neena Gala, Email: ynmvgala@gmail.com

Copyright: () the author(s), publisher and licensee Medip Academy. This is an openaccess article distributed under the terms of the Creative Commons Attribution NonCommercial License, which permits unrestricted noncommercial use, distribution, and reproduction in any medium, provided the original work is properly cited.

\begin{abstract}
Background: From debridement to the appearance of healthy granulation tissue is the maximum time during which the patient suffers more economically and psychologically. Due to high cost expenditure in hospitalisation, dressing material cost and loss of daily earnings during this period which puts a psychological pressure over patient and family especially in India where majority of the population is still below poverty line. Also access to quick treatment is yet not possible to majority of population living in rural with no access to basic healthcare.

Methods: It was an experimental study wherein Wistar rat models were used to check for the wound healing property of Hibiscus Rosa Sinensis (HRS) compared with one of the common standard drugs of treatment for available today, betadine. To observe the wound healing property of the HRS flower extract, the experiment was divided into 2 parts i.e. excision wound model and incision wound model. The groups were treated with respective medication along with a control group of rats.

Results: At the end of the experiment, it was observed that HRS flower extract increased the wound breaking strength as compared to control but not as effectively as compared to the betadine ointment.

Conclusions: HRS flower extract helps in early epithelization and helps in decreasing the wound size. Betadine is associated with adverse events like life threatening allergic reactions, water retention, etc.
\end{abstract}

Keywords: Animal, Betadine, Hibiscus, Skin, Flower, Wound healing, Wistar

\section{INTRODUCTION}

Wound and wound healing is a matter of great concern for all the medical practitioners ever since the dawn of civilization and rise of modern medicine. ${ }^{1}$ Irrespective of the inciting injury, similar healing process takes place, i.e., the lost tissue is not regenerated but is replaced by a fibrous tissue. $^{2}$

Knowledge of wound healing and various promoters of wound healing enable the doctors to manipulate the wound to achieve an optimal result in a rapid period. Infinite literatures on solution of wound healing suggest that the problem is not so simple and straightforward to tackle.

During time of invent of asepsis and dawn of antibiotic era, wound care had achieved milestones, but the widespread development of antibiotic-resistant bacteria is a major roadblock. ${ }^{3}$

In India where majority of population is from poor economic background, it is difficult for many patients to 
afford costly wound care modalities. For these patients being bedridden is a curse as majority of them earn livelihood on daily wages basis. ${ }^{4}$

Pharmaceutical companies are taking advantages of these complexities to introduce multiple remedies, staking high claims for commercial interest. Numerous remedies are available today to counter the various detrimental factors to the natural wound healing process. ${ }^{5}$ Considering these factors, it is necessary to incorporate a simple, cheap, easily available, effective modality that can also be used without any medical supervision to treat wound healing. ${ }^{4}$

Hibiscus rosa sinensis (HRS) extract was found to be such a great modality in better wound care as it is cheap, easily available, had no side effects and can be applied without any assistance. ${ }^{6}$

There are very few studies present in literature comparing the efficacy of HRS extracts with modern medicines. Due to this reason, present study aimed at studying the effectiveness of HRS flower extract as a wound healing agent as compared to betadine ointment.

The aim was to study the wound-healing activity of Hibiscus rosa-sinensis in albino rats. The objective was to evaluate natural wound healing process in (control) group of rats, to evaluate wound healing property of Hibiscus rosa sinensis flower on (test) group of rats, to evaluate wound healing property of betadine topical ointment on (standard) group of rats and to compare the wound healing property of Hibiscus rosa sinensis flower and betadine topical ointment.

\section{METHODS}

The present study was conducted in the Department of Pharmacology, Jawaharlal Nehru Medical College, Sawangi (Meghe), Wardha, Maharashtra, India. The study was carried out for a period of 24 months from November 2016 to November 2018.

The approval from Institutional Animal Ethical Committee of the research protocol was approved by the Institutional Animal Ethical Committee (no: DMIMSDU/IAEC/201617/12), Jawaharlal Nehru Medical College, Sawangi, Meghe, Wardha, Maharashtra, India.

\section{Material}

\section{Hibiscus rosa sinensis (HRS) flower}

Fresh HRS flower were collected from area near by JNMC, Sawangi, Wardha, Maharashtra, India.

\section{Authentication of the plant}

The plants were identified and authenticated by the taxonomist at Mahatma Gandhi Ayurveda College, Wardha, Maharashtra, India.

\section{Purchase of chemicals}

Betadine topical ointment (10\%), ketamine, xylazine and petroleum jelly (ointment base) was obtained from Maharashtra Scientific Medical Store (Wardha, Maharashtra, India).

\section{Animals}

The study was conducted using 36 Wistar Albino rats, of either sex weighing 150-250 g from institutional animal house, Sawangi (Meghe), Wardha, Maharashtra, India.

\section{Inclusion criteria}

- Sex: male and female rats,

- Weight: 150-250 gm,

- $\quad$ Age: 02 months to 14 months.

\section{Exclusion criteria}

- Pregnant female rats,

- Age: more than 14 months,

- Unhealthy/diseased rats.

About 7 grams of reddish-brown semisolid extract was obtained from 50 grams of dried powder of flowers (Figure 1 and Figure 2).

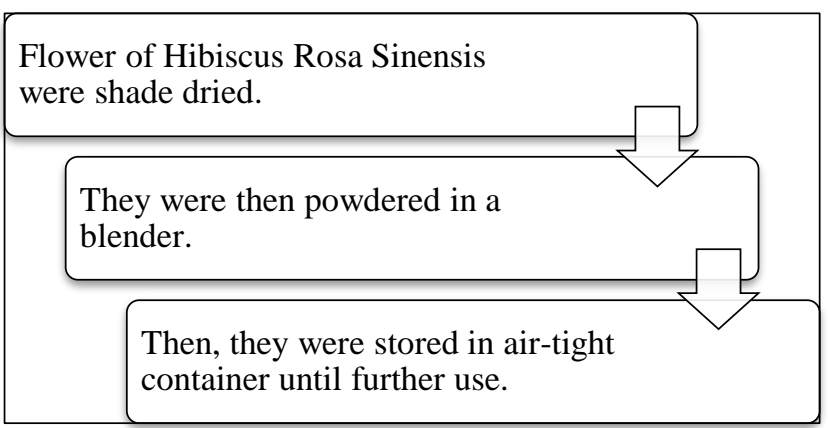

\section{Figure 1: Preparation of plant material in} powdered form.

Powder was extracted in soxhlet apparatus using $95 \%$ ethanol at 60 80 degree celsius for 12 hours.

Extracts were filtered.

Filtrate was then concentrated with a rotatory evaporator to obtain crude extract.

Figure 2: Preparation of crude ethanolic extract. 


\section{Ointment formulations}

About 5\% (w/w) of HRS extract was determined to be the optimum dose to promote wound healing according to Raduan $\mathrm{S}$ et al, and Meena $\mathrm{AK}$ et al. ${ }^{8,9}$ Ointment formulations was prepared from the extract $5 \%(\mathrm{w} / \mathrm{w})$, where 5 gram of the extract was incorporated into 100 grams of petroleum jelly. Betadine topical ointment $(10 \%$ $\mathrm{w} / \mathrm{w}$ ) was used as a standard drug for comparing the wound healing potential of the extract.

\section{Acclimatization}

Rats were only used after 7 day acclimatization period (12:12 hours, light:dark cycle) to the laboratory environment. They were housed under the standard nutritional and environmental conditions of light, temperature and humidity. They were fed with standard laboratory chow and provided water ad libitum. Post experimental study, the rats were returned to the animal house after rehabilitation.

\section{Primary skin irritation test}

The skin irritation test was done by a method based on the test described by Suraj et al. ${ }^{10}$ A $2 \mathrm{~cm}^{2}$ dorsal area was trimmed and then shaved. After cleaning the area with surgical spirit, $5 \% \mathrm{w} / \mathrm{w}$ of extract was applied topically to observe any adverse reaction. Similarly, 10\% w/w betadine ointment was applied topically to observe any adverse reaction. Animals did not show any adverse effects and thus the prepared extract was considered safe for topical application along with the standard drugs.

\section{Preoperative preparation}

Weight of the rats was taken. Pre-emptive analgesia was considered and ophthalmic ointment to the eye was administered following induction of anesthesia to prevent corneal drying. Body hair was removed from the surgical site and surgical scrub alternating between a disinfectant and alcohol was carried out. Warm fluid bag was used to prevent heat loss. Isotonic fluid was kept ready in case of excessive fluid loss.

\section{Induction of anesthesia}

Injection ketamine $(50 \mathrm{mg}): 0.4 \mathrm{ml}$ and injection xylazine $(2 \%)$ : $0.4 \mathrm{ml}$ was given intra-peritoneal. ${ }^{11}$ Since, it is a dissociative anaesthetic, it interrupts the neuronal traffic between the cortex and thalamus. This effect is characterized by sustained reflex movements, stiff muscle, unconsciousness and open eyes. Absence of response to painful stimuli indicates complete induction of anaesthesia.

\section{Post-operative management}

Isotonic fluid was supplied along with warm fluid bag or gloves as required. Rats were frequently checked every 10-
15 mins during recovery from anesthesia. Food and water intake were observed and also analgesic were administered.

\section{Excision wound ${ }^{7}$}

Rats were inflicted with excision wound by cutting away a $500 \mathrm{~mm}^{2}$ full thickness of skin on the back as described by Morton JJ et al, under anaesthesia. ${ }^{12}$

The wound was left undressed to open environment. The rats were divided into following groups and received the following treatment:

Table 1: Grouping for excision wound model.

\begin{tabular}{|c|c|c|}
\hline Group & Total no. of rats & Treatment \\
\hline I (Control) & 6 & Untreated, saline \\
\hline II (Standard) & 6 & $\begin{array}{l}10 \% \mathrm{w} / \mathrm{w} \text { betadine } \\
\text { topical ointment }\end{array}$ \\
\hline III (Test) & 6 & $\begin{array}{l}50 \mathrm{mg} \text { of ointment } \\
\text { prepared from } 5 \% \\
\text { of ethanolic extract } \\
\text { of } H R S\end{array}$ \\
\hline
\end{tabular}

- The ointment was topically applied once a day, starting from the day of the operation, till complete epithelization. This model was used to monitor wound contraction and wound closure time.

- Wound contraction was calculated as percentage reduction in wound area. The progressive change in wound area was monitored by tracing the wound margin on graph paper every alternate day.

Percentage $(\%)$ wound closure $=($ Wound area on Day 0 Wound area on Day 'n')/ (Wound area on Day 0) * 100

\section{Incision wound ${ }^{6,13}$}

After inducing anaesthesia, a $2 \mathrm{~cm}$ long paravertebral incision was made through the full thickness of the skin on left side (flank) of the vertebral column of the rats back as described by Ehrlich HP et al. ${ }^{14}$ The wound was closed with interrupted sutures (ethilion/nylon).

The rats were divided into following groups and received the following treatment:

Table 2: Grouping for incision wound model.

\begin{tabular}{|lll|}
\hline Group & $\begin{array}{l}\text { Total no. } \\
\text { of rats }\end{array}$ & Treatment \\
\hline I (Control) & 6 & Untreated, saline \\
\hline II (Standard) & 6 & $\begin{array}{l}10 \% \mathrm{w} / \mathrm{w} \text { betadine } \\
\text { topical ointment }\end{array}$ \\
\hline III (Test) & 6 & $\begin{array}{l}50 \mathrm{mg} \text { of ointment } \\
\text { prepared from } 5 \% \text { of } \\
\text { ethanolic extract of HRS }\end{array}$ \\
\hline
\end{tabular}


- The ointment was topically applied once a day. The sutures were removed on the 7th day. Woundbreaking strength (wound healing) was measured in anesthetized rats on the 10th day after wounding using a tensiometer.

\section{Measurement of wound breaking strength ${ }^{15}$}

Tensile strength, the force required to open a healing skin wound, was used to measure healing. The instrument used for this measurement is tensiometer. It consisted of animal operation table serving as the base. On one side of the operation table, an IV stand was fixed. It served as a fix point to which one Kelly's forceps was fixed at one end.

Another Kelly's forceps were tied to a piece of fishing line (20-lb test monofilament) to which $500 \mathrm{ml}$ polyethylene bottle was tied at the end. The grips of the Kelly's forceps were then attached to the either side of the incised wound and was to adjust so that the polyethylene bottle was freely hanging in the air.

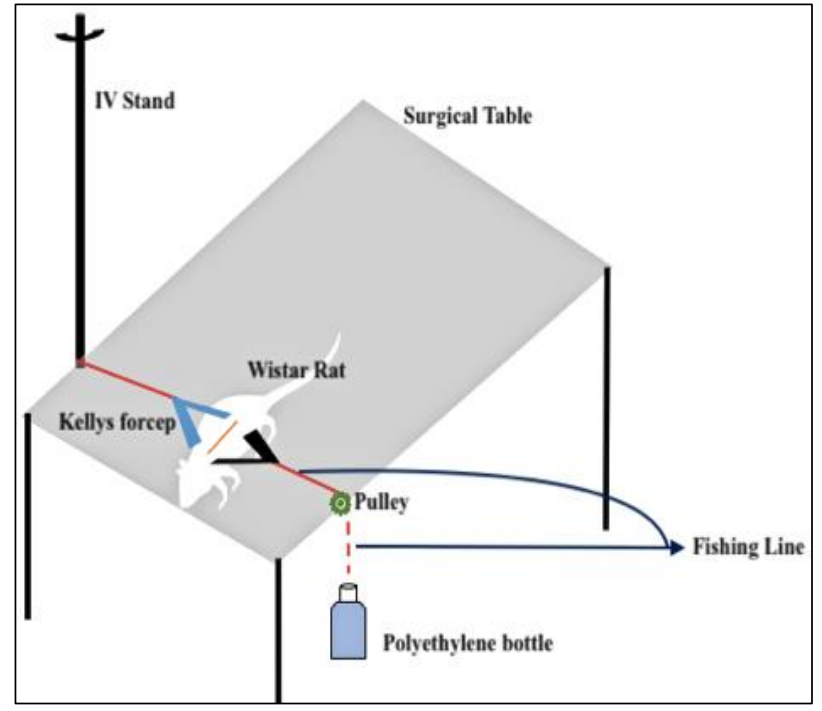

Figure 3: Tensiometer arrangement (self-made).

Table 3: Comparison of wound area $\left(\mathrm{mm}^{2}\right)$ in three groups by applying descriptive statistics.

\begin{tabular}{|c|c|c|c|c|c|c|c|c|c|}
\hline \multirow{2}{*}{ Group } & & \multirow{2}{*}{$\mathbf{N}$} & \multirow{2}{*}{ Mean } & \multirow{2}{*}{ SD } & \multirow{2}{*}{ SE } & \multicolumn{2}{|c|}{$\begin{array}{l}95 \% \text { confidence interval for } \\
\text { mean }\end{array}$} & \multirow{2}{*}{ Minimum } & \multirow{2}{*}{ Maximum } \\
\hline & & & & & & $\begin{array}{l}\text { Lower } \\
\text { bound }\end{array}$ & Upper bound & & \\
\hline \multirow{3}{*}{$\begin{array}{l}\text { Day } \\
0\end{array}$} & Control & 06 & 508.66 & 11.57 & 4.72 & 496.52 & 520.80 & 490.00 & 520.00 \\
\hline & Standard & 06 & 513.66 & 07.73 & 03.15 & 505.54 & 521.78 & 500.00 & 520.00 \\
\hline & Test & 06 & 505.00 & 08.94 & 03.65 & 495.61 & 514.38 & 490.00 & 515.00 \\
\hline \multirow{3}{*}{$\begin{array}{l}\text { Day } \\
2\end{array}$} & Control & 06 & 462.00 & 06.60 & 02.69 & 455.07 & 468.92 & 452.00 & 470.00 \\
\hline & Standard & 06 & 423.16 & 08.40 & 03.42 & 414.35 & 431.98 & 417.00 & 440.00 \\
\hline & Test & 06 & 460.50 & 09.37 & 03.82 & 450.66 & 470.33 & 446.00 & 470.00 \\
\hline \multirow{3}{*}{$\begin{array}{l}\text { Day } \\
4\end{array}$} & Control & 06 & 398.33 & 09.89 & 04.03 & 387.95 & 408.71 & 384.00 & 414.00 \\
\hline & Standard & 06 & 351.83 & 16.22 & 06.62 & 334.80 & 368.86 & 321.00 & 364.00 \\
\hline & Test & 06 & 407.66 & 08.52 & 03.48 & 398.72 & 416.61 & 396.00 & 420.00 \\
\hline \multirow{3}{*}{$\begin{array}{l}\text { Day } \\
6\end{array}$} & Control & 06 & 364.00 & 22.76 & 09.29 & 340.10 & 387.89 & 320.00 & 380.00 \\
\hline & Standard & 06 & 263.16 & 17.64 & 07.20 & 244.64 & 281.68 & 231.00 & 280.00 \\
\hline & Test & 06 & 364.50 & 10.34 & 04.22 & 353.63 & 375.36 & 348.00 & 375.00 \\
\hline \multirow{3}{*}{$\begin{array}{l}\text { Day } \\
8\end{array}$} & Control & 06 & 310.83 & 08.01 & 03.27 & 302.42 & 319.23 & 297.00 & 320.00 \\
\hline & Standard & 06 & 191.66 & 15.95 & 06.51 & 174.91 & 208.41 & 173.00 & 217.00 \\
\hline & Test & 06 & 304.83 & 13.94 & 05.69 & 290.19 & 319.47 & 285.00 & 320.00 \\
\hline \multirow{3}{*}{$\begin{array}{l}\text { Day } \\
10\end{array}$} & Control & 06 & 283.66 & 12.04 & 04.91 & 271.02 & 296.30 & 264.00 & 297.00 \\
\hline & Standard & 06 & 138.66 & 16.76 & 06.84 & 121.07 & 156.26 & 120.00 & 164.00 \\
\hline & Test & 06 & 248.66 & 14.94 & 06.10 & 232.97 & 264.35 & 224.00 & 270.00 \\
\hline \multirow{3}{*}{$\begin{array}{l}\text { Day } \\
12\end{array}$} & Control & 06 & 254.33 & 11.75 & 04.80 & 241.99 & 266.67 & 240.00 & 264.00 \\
\hline & Standard & 06 & 89.16 & 14.20 & 05.79 & 74.26 & 104.07 & 75.00 & 110.00 \\
\hline & Test & 06 & 183.83 & 05.77 & 02.35 & 177.77 & 189.89 & 175.00 & 192.00 \\
\hline \multirow{3}{*}{$\begin{array}{l}\text { Day } \\
14\end{array}$} & Control & 06 & 237.33 & 09.50 & 03.87 & 227.36 & 247.30 & 226.00 & 248.00 \\
\hline & Standard & 06 & 44.50 & 12.38 & 05.05 & 31.49 & 57.50 & 32.00 & 60.00 \\
\hline & Test & 06 & 143.00 & 08.48 & 03.46 & 134.09 & 151.90 & 130.00 & 150.00 \\
\hline \multirow{3}{*}{$\begin{array}{l}\text { Day } \\
16\end{array}$} & Control & 06 & 214.16 & 11.17 & 04.56 & 202.43 & 225.89 & 198.00 & 228.00 \\
\hline & Standard & 06 & 16.83 & 07.85 & 03.20 & 08.58 & 25.08 & 09.00 & 28.00 \\
\hline & Test & 06 & 97.66 & 10.61 & 4.33 & 86.52 & 108.80 & 86.00 & 110.00 \\
\hline \multirow{3}{*}{$\begin{array}{l}\text { Day } \\
18\end{array}$} & Control & 06 & 202.33 & 11.96 & 04.88 & 189.78 & 214.88 & 186.00 & 218.00 \\
\hline & Standard & 06 & 01.66 & 01.96 & 00.80 & -00.39 & 03.73 & 00.00 & 04.00 \\
\hline & Test & 06 & 47.33 & 12.04 & 04.91 & 34.69 & 59.97 & 34.00 & 64.00 \\
\hline
\end{tabular}


Water added to the polyethylene bottle was weighed and considered as the tensile strength of the wound (Figure 3 ).

The endpoint was achieved as soon as the wound gapping was seen. Tensile strength of the healed wound for an individual animal was calculated by the mean determination of the tensile strength of the 2 paravertebral incision.

\section{Statistics}

The data was subjected to statistical evaluation by applying the tests of significance as Paired t-test, One-way ANOVA and multiple comparison test: post hoc Tukey test. The software used in the analysis were SPSS 17.0 version and GraphPad Prism 5.0 and $\mathrm{p}<0.05$ is considered as level of significance.

\section{RESULTS}

Table 3 shows the increasing mean difference of wound area between rats treated with HRS flower extract (test), betadine ointment (standard) and saline treated (control).
On Day 0, the mean wound area of control, standard and test group were $508.66 \pm 11.57 \mathrm{~mm}^{2}, 513.66 \pm 07.73 \mathrm{~mm}^{2}$ and $505.00 \pm 08.94 \mathrm{~mm}^{2}$ respectively. On Day 18 , the readings recorded were $202.33 \pm 11.96 \mathrm{~mm}^{2}, 01.66 \pm 01.96$ $\mathrm{mm}^{2}$ and $47.33 \pm 12.04 \mathrm{~mm}^{2}$ respectively (Table 3).

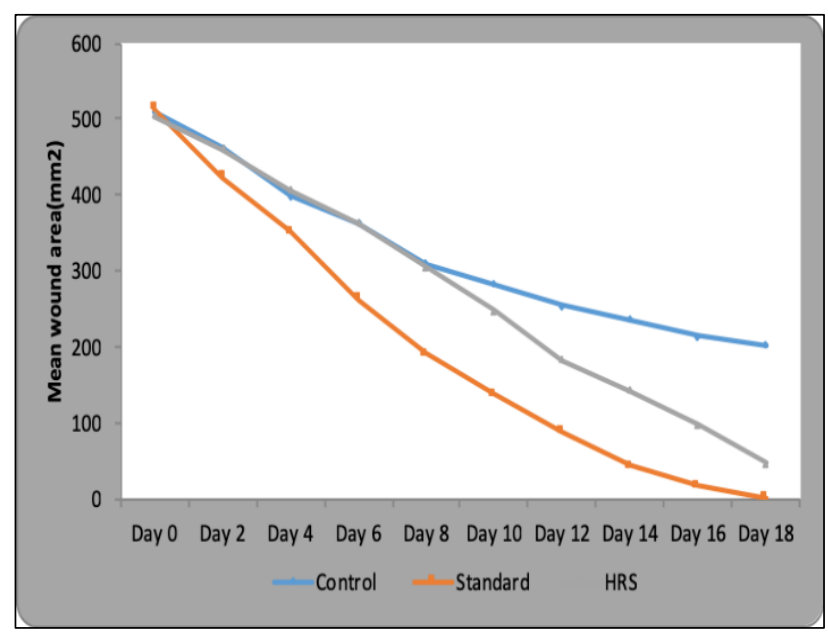

Figure 4: Comparison of wound area $(\mathrm{mm} 2)$ in three groups.

Table 4: Comparison of wound area $\left(\mathrm{mm}^{2}\right)$ in three groups by applying One Way ANOVA.

\begin{tabular}{|c|c|c|c|c|c|c|}
\hline \multicolumn{2}{|c|}{ Source of variations } & Sum of squares & dff & Mean square & $\mathbf{F}$ & p-value \\
\hline \multirow{3}{*}{ Day 0} & Between Groups & 227.11 & 2 & 113.55 & \multirow{3}{*}{1.24} & \multirow{3}{*}{0.316} \\
\hline & Within Groups & 1368.66 & 15 & 91.24 & & \\
\hline & Total & 1595.77 & 17 & & & \\
\hline \multirow{3}{*}{ Day 2} & Between Groups & 5808.11 & 2 & 2904.05 & \multirow{3}{*}{43.11} & \multirow{3}{*}{$0.0001^{*}$} \\
\hline & Within Groups & 1010.33 & 15 & 67.35 & & \\
\hline & Total & 6818.44 & 17 & & & \\
\hline \multirow{3}{*}{ Day 4} & Between Groups & 10733.44 & 2 & 5366.72 & \multirow{3}{*}{37.10} & \multirow{3}{*}{$0.0001^{*}$} \\
\hline & Within Groups & 2169.50 & 15 & 144.63 & & \\
\hline & Total & 12902.94 & 17 & & & \\
\hline \multirow{3}{*}{ Day 6} & Between Groups & 40872.11 & 2 & 20436.05 & \multirow{3}{*}{65.44} & \multirow{3}{*}{$0.0001 *$} \\
\hline & Within Groups & 4684.33 & 15 & 312.28 & & \\
\hline & Total & 45556.44 & 17 & & & \\
\hline \multirow{3}{*}{ Day 8} & Between Groups & 54086.77 & 2 & 27043.38 & \multirow{3}{*}{158.02} & \multirow{3}{*}{$0.0001 *$} \\
\hline & Within Groups & 2567.00 & 15 & 171.13 & & \\
\hline & Total & 56653.77 & 17 & & & \\
\hline \multirow{3}{*}{ Day 10} & Between Groups & 68700.00 & 2 & 34350.00 & \multirow{3}{*}{158.63} & \multirow{3}{*}{$0.0001 *$} \\
\hline & Within Groups & 3248.00 & 15 & 216.53 & & \\
\hline & Total & 71948.00 & 17 & & & \\
\hline \multirow{3}{*}{ Day 12} & Between Groups & 82424.11 & 2 & 41212.05 & \multirow{3}{*}{331.10} & \multirow{3}{*}{$0.0001 *$} \\
\hline & Within Groups & 1867.00 & 15 & 124.46 & & \\
\hline & Total & 84291.11 & 17 & & & \\
\hline \multirow{3}{*}{ Day 14} & Between Groups & 111571.44 & 2 & 55785.72 & \multirow{3}{*}{530.00} & \multirow{3}{*}{$0.0001 *$} \\
\hline & Within Groups & 1578.83 & 15 & 105.25 & & \\
\hline & Total & 113150.27 & 17 & & & \\
\hline \multirow{3}{*}{ Day 16} & Between Groups & 118093.44 & 2 & 59046.72 & \multirow{3}{*}{591.65} & \multirow{3}{*}{$0.0001 *$} \\
\hline & Within Groups & 1497.00 & 15 & 99.80 & & \\
\hline & Total & 119590.44 & 17 & & & \\
\hline Day 18 & Between Groups & 132755.11 & 2 & 66377.55 & 681.96 & $0.0001 *$ \\
\hline
\end{tabular}


From Figure 4, it is evident that betadine had the most efficacious wound healing activity followed by the HRS flower extract (Figure 4). The difference in the wound healing property observed in comparing the 3 groups was statistically significant (p-value $=0.0001) \quad$ (Table 4 and Table 5).
Table 6 illustrates the wound area over a period of 18 days. While the standard group shows the most effective healing, the test group shows a slow but effective healing as well over a period of 18 days.

Table 5: Comparison of wound area $\left(\mathrm{mm}^{2}\right)$ in three groups by applying multiple comparisons: post hoc Tukey test.

\begin{tabular}{|c|c|c|c|c|c|c|c|}
\hline \multirow{2}{*}{$\begin{array}{l}\text { Time } \\
\text { period }\end{array}$} & \multirow{2}{*}{ Group } & & \multirow{2}{*}{$\begin{array}{l}\text { Mean } \\
\text { diff. (I-J) }\end{array}$} & \multirow{2}{*}{ Std. error } & \multirow{2}{*}{ p-value } & \multicolumn{2}{|c|}{$95 \%$ confidence interval } \\
\hline & & & & & & Lower bound & Upper bound \\
\hline \multirow{3}{*}{ Day 0} & \multirow{2}{*}{ Control } & Standard & -5.00 & 5.51 & 0.645 & -19.32 & 9.32 \\
\hline & & Test & 3.66 & 5.51 & 0.787 & -10.65 & 17.99 \\
\hline & Standard & Test & 8.66 & 5.51 & 0.288 & -5.65 & 22.99 \\
\hline \multirow{3}{*}{ Day 2} & \multirow{2}{*}{ Control } & Standard & 38.83 & 4.73 & $0.0001 *$ & 26.52 & 51.14 \\
\hline & & Test & 1.50 & 4.73 & 0.946 & -10.80 & 13.80 \\
\hline & Standard & Test & -37.33 & 4.73 & $0.0001 *$ & -49.64 & -25.02 \\
\hline \multirow{3}{*}{ Day 4} & \multirow{2}{*}{ Control } & Standard & 46.50 & 6.94 & $0.0001 *$ & 28.46 & 64.53 \\
\hline & & Test & -9.33 & 6.94 & 0.394 & -27.36 & 8.70 \\
\hline & Standard & Test & -55.83 & 6.94 & $0.0001 *$ & -73.86 & -37.79 \\
\hline \multirow{3}{*}{ Day 6} & \multirow{2}{*}{ Control } & Standard & 100.83 & 10.20 & $0.0001 *$ & 74.33 & 127.33 \\
\hline & & Test & -0.50 & 10.20 & 0.999 & -27.00 & 26.00 \\
\hline & Standard & Test & -101.33 & 10.20 & $0.0001 *$ & -127.83 & -74.8 \\
\hline \multirow{3}{*}{ Day 8} & \multirow{2}{*}{ Control } & Standard & 119.16 & 7.55 & $0.0001 *$ & 99.54 & 138.78 \\
\hline & & Test & 6.00 & 7.55 & 0.712 & -13.61 & 25.61 \\
\hline & Standard & Test & -113.16 & 7.55 & $0.0001 *$ & -132.78 & -93.54 \\
\hline \multirow{3}{*}{ Day 10} & \multirow{2}{*}{ Control } & Standard & 145.00 & 8.49 & $0.0001 *$ & 122.93 & 167.06 \\
\hline & & Test & 35.00 & 8.49 & $0.002 *$ & 12.93 & 57.06 \\
\hline & Standard & Test & -110.00 & 8.49 & $0.0001 *$ & -132.06 & -87.93 \\
\hline \multirow{3}{*}{ Day 12} & \multirow{2}{*}{ Control } & Standard & 165.16 & 6.44 & $0.0001 *$ & 148.43 & 181.89 \\
\hline & & Test & 70.50 & 6.44 & $0.0001 *$ & 53.76 & 87.2308 \\
\hline & Standard & Test & -94.66 & 6.44 & $0.0001 *$ & -111.39 & -77.93 \\
\hline \multirow{3}{*}{ Day 14} & \multirow{2}{*}{ Control } & Standard & 192.83 & 5.92 & $0.0001 *$ & 177.44 & 208.21 \\
\hline & & Test & 94.33 & 5.92 & $0.0001 *$ & 78.94 & 109.71 \\
\hline & Standard & Test & -98.50 & 5.92 & $0.0001 *$ & -113.8 & -83.11 \\
\hline \multirow{3}{*}{ Day 16} & \multirow{2}{*}{ Control } & Standard & 197.33 & 5.76 & $0.0001 *$ & 182.35 & 212.3 \\
\hline & & Test & 116.50 & 5.76 & $0.0001 *$ & 101.51 & 131.48 \\
\hline & Standard & Test & -80.83 & 5.76 & $0.0001 *$ & -95.81 & -65.85 \\
\hline \multirow{3}{*}{ Day 18} & \multirow{2}{*}{ Control } & Standard & 200.66 & 5.69 & $0.0001 *$ & 185.87 & 215.46 \\
\hline & & Test & 155.00 & 5.69 & $0.0001 *$ & 140.20 & 169.79 \\
\hline & Standard & Test & -45.66 & 5.69 & $0.0001 *$ & -60.46 & -30.87 \\
\hline
\end{tabular}

Table 6: Percentage of wound healing.

\begin{tabular}{|llll|}
\hline Day (Duration) & Control & Standard & Test \\
\hline Day 0 & $0 \%$ & $0 \%$ & $0 \%$ \\
\hline Day 2 & $9 \%$ & $18 \%$ & $9 \%$ \\
\hline Day 4 & $22 \%$ & $32 \%$ & $19 \%$ \\
\hline Day 6 & $28 \%$ & $49 \%$ & $28 \%$ \\
\hline Day 8 & $39 \%$ & $63 \%$ & $40 \%$ \\
\hline Day 10 & $44 \%$ & $73 \%$ & $51 \%$ \\
\hline Day 12 & $50 \%$ & $83 \%$ & $64 \%$ \\
\hline Day 14 & $53 \%$ & $91 \%$ & $72 \%$ \\
\hline Day 16 & $58 \%$ & $97 \%$ & $81 \%$ \\
\hline Day 18 & $60 \%$ & $100 \%$ & $91 \%$ \\
\hline
\end{tabular}


Table 7: Comparison of skin breaking strength (g) in three groups by applying descriptive statistics.

\begin{tabular}{|c|c|c|c|c|c|c|c|c|}
\hline \multirow[t]{2}{*}{ Groups } & \multirow[t]{2}{*}{$\mathbf{N}$} & \multirow[t]{2}{*}{ Mean } & \multirow[t]{2}{*}{ SD } & \multirow{2}{*}{$\begin{array}{l}\text { Std. } \\
\text { Error }\end{array}$} & \multicolumn{2}{|c|}{$\begin{array}{l}95 \% \text { Confidence Interval for } \\
\text { Mean }\end{array}$} & \multirow[t]{2}{*}{ Minimum } & \multirow[t]{2}{*}{ Maximum } \\
\hline & & & & & Lower Bound & Upper Bound & & \\
\hline Control & 06 & 15.76 & 1.12 & 0.46 & 14.58 & 16.94 & 14.00 & 17.00 \\
\hline Standard & 06 & 41.36 & 2.04 & 0.83 & 39.22 & 43.51 & 38.00 & 44.00 \\
\hline Test & 06 & 26.50 & 2.16 & 0.88 & 24.22 & 28.77 & 24.00 & 30.00 \\
\hline
\end{tabular}

Table 8: Comparison of skin breaking strength (g) in three groups by applying One-way ANOVA.

\begin{tabular}{|llllll|}
\hline Source of variations & Sum of Squares & df & Mean Square & F & p-value \\
\cline { 1 - 4 } Between Groups & 1983.16 & 2 & 991.58 & & \\
\cline { 1 - 4 } Within Groups & 50.76 & 15 & 3.38 & & $0.0001^{*}$ \\
\cline { 1 - 5 } Total & 2033.93 & 17 & & & \\
\hline
\end{tabular}

Table 9: Comparison of skin breaking strength (g) in three groups by applying multiple comparisons: post hoc Tukey Test.

\begin{tabular}{|lllllll|}
\hline \multirow{2}{*}{ Groups } & & $\begin{array}{l}\text { Mean Difference } \\
\text { (I-J) }\end{array}$ & Std. Error & p-value & \multicolumn{2}{c|}{ 95\% Confidence Interval } \\
\hline \multirow{2}{*}{ Control } & Standard & -25.60 & 1.06 & $0.0001 *$ & -28.35 & -22.84 \\
\cline { 2 - 7 } & Test & -10.73 & 1.06 & $0.0001^{*}$ & -13.49 & -7.97 \\
\hline Standard & Test & 14.86 & 1.06 & $0.0001 *$ & 12.10 & 17.62 \\
\hline
\end{tabular}

Percentage reduction in wound area recorded on Day 18 in control, standard and test were $60 \%, 100 \%$ and $91 \%$ respectively (Table 6).

Table 7 and Figure 5 shows the comparison of skin breaking strength observed in the three groups. The skin breaking strength of control, standard and test group were $15.76 \pm 1.12 \mathrm{~g}, 41.36 \pm 2.04 \mathrm{~g}$ and $26.50 \pm 2.16 \mathrm{~g}$ respectively. The group of rats treated with betadine topical ointment showed the highest tensile strength followed by the HRS flower extract (Table 7) (Figure 5).

The difference in the skin breaking strength observed in comparing the 3 groups was statistically significant (pvalue $=0.0001)$ (Table 8 and Table 9).

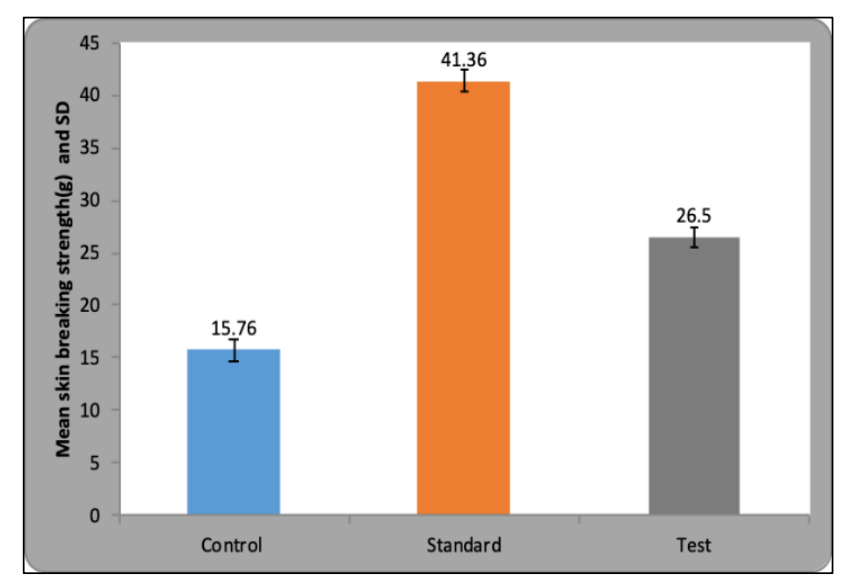

Figure 5: Comparison of skin breaking strength (g) in three groups.

\section{DISCUSSION}

The present study was undertaken to evaluate wound healing activity of Hibiscus rosa sinensis linn in albino rats and comparing it with standard treatment. This was considered as the aim of the study. The finding and results from this study is similar to several other studies that have included extracts of HRS flower. . $^{6,13,16}$

Betadine is a formulation based on povidone-iodine. Povidone iodine is an effective antiseptic with bactericidal property against gram positive and gram-negative organism, that does not impede wound healing. ${ }^{17-20}$ However, several studies have reported side effects such as life-threatening allergic reactions, visible water retention, boy temperature fluctuations etc. ${ }^{21}$

Bhaskar A et al, had reported similar findings regarding the skin breaking strength in their study by the $12^{\text {th }}$ day. ${ }^{13}$

Shivananda NB et al, reported higher collagen content in HRS flower treated rats. They demonstrated an increase in hydroxyproline, an amino acid which is a major component of collagen. ${ }^{6}$

Although HRS flower as such has no specific antimicrobial activity of its own, the wound healing promoting effect can be attributed to its various hormonal activities which include hypoglycaemic, androgen like, antioxidant and anticonvulsant activities. ${ }^{6}$ The increased tensile strength, enhanced wound contraction and short epithelization period clearly indicate and support the wound healing promoting effect of HRS flower extract. 
Shen $\mathrm{H}$ et al, demonstrated that N-butyl alcohol extract of HRS (NHRS) flower is far more potent than other forms of HRS extract. They also suggested that NHRS increases secretion of vascular endothelial growth factor (VEGF) which accelerates wound healing and accelerates the regeneration of epidermal layer by upregulation of transforming growth factor (TGF- $\beta 1$ ). They concluded that NHRS elevates levels of VEGF and TGF-B1 which promotes faster wound repair via increased angiogenesis, collagen fibre deposition and as well as increasing the activity of macrophages. ${ }^{16}$

Bhaskar A et al, reported the presence of tannins, saponins, flavonoids and terpenoids in their phytochemical evaluation of HRS flower extract which highlighting flavonoids as the active substance in promoting wound healing. However, they have reported an increase in the hexosamine and uronic acid molecules which are responsible for the synthesis of extracellular matrix as the primary method of wound healing. ${ }^{13}$

Factors that delay the healing example diabetes, obesity, stress, and other are not taken purposefully to establish that HRS improves wound healing when no other deteriorating factors are present.

\section{Recommendations}

The experiment needs further evaluation in other animals such as dogs, monkeys etc. Clinical evaluation needs to be carried out. Cellular level and molecular level studies are required to conclude regarding mechanism of action.

\section{ACKNOWLEDGEMENTS}

Authors would like to thank Department of Pharmacology, Animal House, Central Research Laboratory and Animal Ethical committee of Jawaharlal Nehru Medical College, Sawangi, Wardha, Maharashtra, India, Mahatma Gandhi Ayurveda College, Selu, Maharashtra, Indian Council of Medical Research, New Delhi, India for support during study.

Funding: No funding sources Conflict of interest: None declared

Ethical approval: The study was approved by the Institutional Animal Ethical Committee, Jawaharlal Nehru Medical College, Sawangi, Meghe, Wardha, Maharashtra, India (no: DMIMSDU/IAEC/2016-17/12)

\section{REFERENCES}

1. Forrest RD. Early history of wound treatment. J R Soc Med. 1982;75(3):198-205.

2. Shield HealthCare. How wounds heal: the 4 main phases of wound healing, 2015. Available at: http://www.shieldhealthcare.com/community/wound/ 2015/12/18/how-wounds-heal-the-4-main-phases-ofwound-healing/. Accessed 21 September 2018.
3. Boateng JS, Matthews KH, Stevens HNE, Eccleston GM. Wound healing dressings and drug delivery systems: a review. J Pharm Sci. 2008;97(8):2892-923.

4. Gupta N, Gupta SK, Shukla VK, Singh SP. An Indian community-based epidemiological study of wounds. J Wound Care. 2004;13(8):323-5.

5. Daunton C, Kothari S, Smith L, Steele D. A history of materials and practices for wound management. Wound Pract Res. 2012;20:174-86.

6. Shivananda NB, Raju SS, Orette FA, Rao CAV. Effects of Hibiscus rosa sinensis L (Malvaceae) on wound healing activity: a preclinical study in a sprague dawley rat. Int $\mathrm{J}$ Low Extrem Wounds. 2007;6(2):76-81.

7. Bhaskar A, Nithya V. Evaluation of the woundhealing activity of Hibiscus rosa sinensis $L$ (Malvaceae) in Wistar albino rats. Indian J Pharmacol. 2012;44(6):694-8.

8. Meena AK, Jain A, Pandey K, Singh RK. Acute toxicity and genotoxic activity of Hibiscus rosasinensis flower extract. Am J Phytomed Clin Therapeutics. 2014;2(4):524-9.

9. Raduan SZ, Abdul Aziz MW, Roslida AH, Zakaria ZA, Zuraini A, Hakim MN. Anti-inflammatory effects of Hibiscus rosa-sinensis L. and Hibiscus rosa-sinensis var. alba ethanol extracts. Int J Pharm Pharmaceut Sci. 2013;5(4):754-62.

10. Davis SV, Shenoi SD, Prabhu S, Shirwaiker A, Balachandran C. Clinical evaluation of patients patch tested with plant series: a prospective study. Indian J Dermatol. 2011;56(4):383-8.

11. Injectable Anesthesia. Research at Penn State. Available at: https://www.research.psu.edu/arp/anesthesia/injectabl e-anesthesia.html. Accessed 27 August 2018.

12. Morton JJ, Malone MH. Evaluation of vulneray activity by an open wound procedure in rats. Arch Int Pharmacodyn Ther. 1972;196(1):117-26.

13. Bhaskar A, Nithya V. Evaluation of the woundhealing activity of Hibiscus rosa sinensis $L$ (Malvaceae) in Wistar albino rats. Ind J Pharmacol. 2012;44(6):694-8.

14. Ehrlich HP, Hunt TK. Effects of cortisone and vitamin A on wound healing. Ann Surg. 1968;167(3):324-8.

15. Lodhi S, Jain AP, Rai G, Yadav AK. Preliminary investigation for wound healing and antiinflammatory effects of Bambusa vulgaris leaves in rats. J Ayurveda Integr Med. 2016;7(1):14-22.

16. Shen HM, Chen C, Jiang JY, Zheng YL, Cai WF, Wang B, et al. The N-butyl alcohol extract from Hibiscus rosa-sinensis $L$. flowers enhances healing potential on rat excisional wounds. J Ethnopharmacol. 2017;198:291-301.

17. Bigliardi PL, Alsagoff SAL, El-Kafrawi HY, Pyon J$\mathrm{K}$, Wa CTC, Villa MA. Povidone iodine in wound healing: a review of current concepts and practices. Int J Surg. 2017;44:260-8.

18. Burks RI. Povidone-iodine solution in wound treatment. Phys Ther. 1998;78(2):212-8. 
19. Durani P, Leaper D. Povidone-iodine: use in hand disinfection, skin preparation and antiseptic irrigation. Int Wound J. 2008;5(3):376-87.

20. Fleischer W, Reimer K. Povidone-iodine in antisepsisstate of the art. Dermatol. 1997;195(2):3-9.

21. McDowell S. Are we using too much Betadine? RN. 1991;54(7):43-5.
Cite this article as: Gala MYN, Pathak SS.

Evaluation of wound healing activity of Hibiscus rosa sinensis linn in albino rats. Int $\mathbf{J}$ Basic Clin

Pharmacol 2019;8:1303-11. 